\title{
GLADE: A Galaxy Catalogue for Multi-Messenger Searches in the Advanced Gravitational-Wave Detector Era
}

\author{
G. Dálya ${ }^{1,2 \star}$, G. Galgóczi ${ }^{1,2}$, L. Dobos $^{1}$, Z. Frei ${ }^{1,2}$, I. S. Heng ${ }^{3}$, R. Macas ${ }^{4}$, \\ C. Messenger ${ }^{3}$, P. Raffai ${ }^{1,2}$ and R. S. de Souza ${ }^{5}$ \\ ${ }^{1}$ Institute of Physics, Eötvös University, 1117 Budapest, Hungary \\ ${ }^{2}$ MTA-ELTE Astrophysics Research Group, 1117 Budapest, Hungary \\ ${ }^{3}$ SUPA, University of Glasgow, Glasgow G12 8QQ, United Kingdom \\ ${ }^{4}$ School of Physics and Astronomy, Cardiff University, Cardiff CF24 3AA, United Kingdom \\ ${ }^{5}$ Department of Physics 8 Astronomy, University of North Carolina at Chapel Hill, Chapel Hill, NC 27599-3255, USA
}

Accepted XXX. Received YYY; in original form ZZZ

\begin{abstract}
We introduce a value-added full-sky catalogue of galaxies, named as Galaxy List for the Advanced Detector Era, or GLADE. The purpose of this catalogue is to (i) help identifications of host candidates for gravitational-wave events, (ii) support target selections for electromagnetic follow-up observations of gravitational-wave candidates, (iii) provide input data on the matter distribution of the local universe for astrophysical or cosmological simulations, and (iv) help identifications of host candidates for poorly localised electromagnetic transients, such as gamma-ray bursts observed with the InterPlanetary Network. Both being potential hosts of astrophysical sources of gravitational waves, GLADE includes inactive and active galaxies as well. GLADE was constructed by cross-matching and combining data from five separate (but not independent) astronomical catalogues: GWGC, 2MPZ, 2MASS XSC, HyperLEDA and SDSS-DR12Q. GLADE is complete up to $d_{L}=37_{-4}^{+3} \mathrm{Mpc}$ in terms of the cumulative $B$-band luminosity of galaxies within luminosity distance $d_{L}$, and contains all of the brightest galaxies giving half of the total $B$-band luminosity up to $d_{L}=91 \mathrm{Mpc}$. As $B$-band luminosity is expected to be a tracer of binary neutron star mergers (currently the prime targets of joint GW+EM detections), our completeness measures can be used as estimations of completeness for containing all binary neutron star merger hosts in the local universe.
\end{abstract}

Key words: catalogues - galaxies: distances and redshifts.

\section{INTRODUCTION}

Advanced LIGO (aLIGO, see Aasi et al. 2015) and Advanced Virgo (AdV, see Acernese et al. 2015) are secondgeneration gravitational-wave (GW) detectors located in Hanford, US-WA, Livingston, US-LA, and near Cascina, Italy, respectively. The two aLIGO detectors began their second observing run (O2) on 30 November 2016, with an improved network sensitivity compared to the first observing run (O1, during which the first detections of GWs from binary black hole mergers were accomplished, see Abbott et al. 2016a). AdV joined the network of aLIGO detectors during O2, on 1 August 2017 (Abbott et al. 2017a).

An ongoing effort of the emergent field of multimessenger astronomy (see e.g. Chassande-Mottin et al.

* E-mail: dalyag@caesar.elte.hu (DG);
2011 ) is to discover electromagnetic (EM) counterparts of transient GW events with targeted EM follow-up observations (Abbott et al. 2016b). Maps of posterior probability densities for sky positions of GW source candidates are being distributed by the LIGO-Virgo Collaboration to astronomer partners, who then carry out follow-up observations at various EM wavelengths from radio to gamma bands (see e.g. Abbott et al. 2017b, Aab et al. 2016, Abbott et al. 2016c, Cowperthwaite et al. 2016).

The 90 percent credible localisation areas of transient GW sources produced with the two aLIGO detectors currently cover hundreds of square degrees (Abbott et al. 2016b) (the exact size depends on the source location relative to the detectors, and the signal-to-noise ratio of the signal, see e.g. Bécsy et al. 2017, Berry et al. 2015, and Essick et al. 2015), which is at least an order of magnitude larger than the field of view of most of the EM follow-up 


\section{Dálya, Galgóczi, Dobos, Frei, Heng, Macas, Messenger, Raffai \& de Souza}

instruments. This makes the identification of EM counterparts challenging even with wide-field telescopes, due to the large number of pointings and long integration times required for follow-ups, as well as due to the false positives potentially obtained from these observations. Because of this, the optimization of EM follow-up observing strategies is an important task and a widely researched topic, discussed in details in e.g. Abadie et al. (2012a), Aasi et al. (2014a), Singer et al. (2014), Gehrels et al. (2016) and Coughlin et al. (2018). Since in the upcoming years more GW detectors, such as KAGRA (Somiya 2012) and LIGOIndia (Iyer at al. 2011), are coming online, GW source localisations are expected to greatly improve. For example, it is estimated that more than 20 percent of the 90 percent credible localisation areas will have sizes smaller than 5 square degrees when produced by the 4-detector network of aLIGO, $\mathrm{AdV}$, and LIGO-India in 2024 (Abbott et al. 2016b).

Joint detections of temporally coincident GWs and high-energy neutrinos (HEN) would also present multiple advantages compared to the detection of GWs or HENs only, such as increased search sensitivity, or introducing constrains on the population of astrophysical GW+HEN sources (Baret et al. 2012). The distribution of potential sources, inferred from galaxy catalogues, can be used in joint GW+HEN searches to increase sensitivity and reject false coincidences.

Binary neutron star (BNS) coalescences followed by possible kilonova emissions (see e.g. Li \& Paczyński 1998, Metzger et al. 2010, Tanvir et al. 2013) are currently the most promising sources for joint GW and EM observations. The network of aLIGO and AdV detectors achieved the detection of such an event on August 17, 2017 (Abbott et al. 2017 c), which lead to the discovery of the EM counterpart in various $\mathrm{EM}$ bands and to the identification of the host galaxy (Abbott et al. 2017b), partially with the help of the catalogue presented here (see Section 4 for details). Kilonovae have observable EM emissions lasting for $\gtrsim 1$ week in near infrared. This is a reasonable time frame after a GW detection to search through galaxies from an existing catalogue for a fading EM counterpart, but can be too short to cover the whole localisation area with EM observations deep enough to achieve the detection of the EM counterpart. Furthermore, extending such an existing galaxy catalogue with rapid galaxy surveys within the GW localisation areas, carried out by dedicated small telescopes, is also feasible in this timescale (Bartos et al. 2015).

As it has been shown in multiple papers (e.g. Abadie et al. 2012b, Hanna et al. 2014), using galaxy catalogues in target selections for follow-up observations greatly increases the chance of detecting the EM counterpart, even if the catalogue is incomplete. Since we expect GW sources to reside in or near galaxies, restricting follow-up observations to galaxies can decrease significantly the required number of pointings, the total integration time, and the number of false positives found. Galaxies inside the localisation volume (see e.g. Del Pozzo et al. 2018 and Singer et al. 2016) of a GW event can be ranked in terms of probability of hosting a BNS detected by GW detectors, based on e.g. their B-band luminosities (see Hanna et al. 2014 for details), and astronomers can observe these host candidates in a sequential order from high to low probabilities, thereby enhancing the likelihood for EM counterpart detection within a more limited time.
Alternative applications of a galaxy catalogue include identifying host candidates for poorly localised EM transients, see e.g. Fan et al. (2014). For example, there have been several searches for GW signals associated with gamma-ray bursts (GRBs) using data from the LIGO and Virgo detectors (see e.g. Aasi et al. 2014b). By using the time and sky location information from EM observations, the parameter space of the GW search can be significantly reduced, which improves the efficiency of the GW search. A galaxy catalogue can be used to identify potential host galaxies for poorly localised (e.g. by the InterPlanetary Network or IPN, see Hurley et al. 2013) GRBs, that can be of particular interest for the GW community if the GRB error box or its close proximity contains galaxies that reside within the horizon of advanced GW detectors (Abbott et al. 2016d). Using the results from the GW search and the distance information of the host candidates, we can derive implications on source models, potentially leading to rejecting some of the host candidates or even some of the source models. A galaxy catalogue with high completeness can be useful for astrophysical and cosmological simulations as well, providing prior information on the matter distribution of the local universe (see e.g. Soneira \& Peebles 1978). Galaxy catalogues will be important for statistical cosmological inference as suggested by Schutz (1986) and Del Pozzo (2012).

As models suggest that the number of possible sources of coincident GW and EM emission in a galaxy is traced by the total $B$-band luminosity of the galaxy (through active star formation, see e.g. Phinney 1991), a galaxy catalogue supporting follow-up searches should be as complete as possible in terms of the cumulative $B$-band luminosity of its galaxies (Baret et al. 2012). Note however, that according to Fong et al. (2013) a quarter of short GRBs (produced by BNS mergers, see e.g. Tanvir et al. 2013) occur in elliptical galaxies, that have low star forming rates, and thus low $B$-band luminosities. This suggests that BNS merger rates for individual galaxies are better estimated by a weighed combination of the galaxies' $B$-band luminosities and stellar masses, where the weighing can depend on the galaxies' morphological types.

In the initial detector era, several EM follow-up partners used the Gravitational Wave Galaxy Catalog (GWGC, see White et al. 2011) for observational target selections. This catalogue contains galaxies only within $\sim 100 \mathrm{Mpc}$, and with the since-updated distance data for its entries GWGC is only complete within $\sim 30 \mathrm{Mpc}$ in terms of cumulative $B$-band luminosity, with the completeness falling rapidly at greater distances (see Section 3 for details). During the initial detector era, the BNS ranges ${ }^{1}$ for the individual GW detectors did not exceed $\sim 30 \mathrm{Mpc}$ (Abadie et al. 2012c), and thus GWGC served the EM target selections well in this past era. Advanced GW detectors have larger ranges for BNS coalescences, which can be further extended if multiple detectors operate as a coherent network in GW searches. As

1 The BNS range is the average distance from which a GW detector can detect a circular binary of two $1.4 M_{\odot}$ neutron stars with a signal-to-noise ratio of 8 , where the average is calculated over all possible sky positions and orbital inclinations (Abbott et al. 2016b). Note that the maximum distance from which a BNS (i.e. with optimal sky direction and inclination) can be detected is $\sim 2.26$ times larger (Finn \& Chernoff 1993). 
a result, more distant galaxies, even at $\sim 300 \mathrm{Mpc}$ or further (Evans et al. 2016) can be potential hosts of detectable GW+EM sources, and therefore should be included in a galaxy catalogue that aims to support EM follow-up efforts.

We have constructed a galaxy catalogue called Galaxy List for the Advanced Detector Era (GLADE) in order to support the previously described EM follow-up efforts, and to meet the challenges imposed on galaxy catalogues by the improved sensitivities of advanced GW detectors. Both being potential hosts of astrophysical sources of GWs, GLADE includes inactive and active galaxies as well (where the fact that a GLADE object is an active galaxy hosting a quasar is indicated in the catalogue). The aim of this paper is to describe the construction and properties of GLADE, as well as to point out the applicability of it in target selections for EM follow-up observations, and in other selected areas.

This paper is organized as follows. In Section 2, we introduce methods and catalogues used for constructing GLADE. In Section 3, we describe the completeness of GLADE with two different methods that use the measured $B$-band luminosities of galaxies and that allows comparisons of the completeness of GLADE with that of other existing full-sky catalogues' (e.g. GWGC, and the Census of the Local Universe or CLU presented in Gehrels et al. 2016). In Section 4 , we present how GLADE is already in use for identifying host candidates of GW events by multiple collaborations. Furthermore, we suggest applications of GLADE beyond GW-triggered EM observations, including one where we use GLADE in an automated process to identify potential host galaxies for poorly localised GRBs detected by the IPN. Finally, in Section 5, we summarize our conclusions and outline future plans for improving GLADE in terms of completeness and accuracy of its parameters.

Throughout this paper we adopt a flat $\Lambda \mathrm{CDM}$ cosmology with the following parameters: $H_{0}=100 \mathrm{~h}=$ $70 \mathrm{~km} \mathrm{~s}^{-1} \mathrm{Mpc}^{-1}, \Omega_{\mathrm{M}}=0.27$ and $\Omega_{\Lambda}=0.73$.

\section{CATALOGUE COMPILATION AND STATISTICS}

In the construction of GLADE, we started with crossmatching five separate (but not independent) astronomical catalogues: the GWGC $^{2}$ (White et al. 2011), the HyperLEDA catalogue $^{3}$ (Makarov et al. 2014), the 2 Micron All-Sky Survey Extended Source Catalog ${ }^{4}$ (2MASS XSC, see Skrutskie et al. 2006), the 2MASS Photometric Redshift Catalog 5 (2MPZ, see Bilicki et al. 2014), and the Sloan Digital Sky Survey quasar catalogue from the 12th data release ${ }^{6}$ (SDSS-DR12Q, see Pâris et al. 2016). In this section, we describe the relevant characteristics of these five catalogues, and discuss the cross-matching method we applied and results we obtained.

GWGC is a catalogue of $\sim 50,000$ galaxies and $\sim 150$ globular clusters, that is a result of merging three existing catalogues: the Tully Nearby Galaxy Catalog (Tully 1987),

\footnotetext{
2 vizier.u-strasbg.fr/viz-bin/VizieR?-source=GWGC

3 leda.univ-lyon1.fr/

4 ipac.caltech.edu/2mass/

5 ssa.roe.ac.uk/TWOMPZ.html

6 sdss.org/dr12/algorithms/boss-dr12-quasar-catalog
}

the Catalog of Neighboring Galaxies (Karachentsev et al. 2004), and the V8k catalogue (Tully et al. 2009). In the construction of GWGC, authors of White et al. (2011) used the HyperLEDA catalogue to provide supplemental data (e.g. position angles) for objects where such was available. GWGC does not contain galaxies beyond luminosity distance $d_{L} \simeq 100 \mathrm{Mpc}$ due to various recession velocity cuts applied in the catalogues GWGC has been created from. GWGC includes $B$ magnitude and luminosity distance data, along with their errors, for nearly all its entries. Luminosity distances were derived from distance measurements that used a variety of methods, each having had their own measurement errors within the range of 10 to 20 percent. $B$ magnitudes in GWGC have an average error of $\Delta B=0.37^{m}$. We found that $B$ magnitude values of globular clusters in GWGC were not reliable, therefore we have corrected them using the newest data from the VizieR database ${ }^{7}$ (Ochsenbein et al. 2000).

HyperLEDA is a catalogue of over 3 million objects, created by merging the LEDA (Lyon-Meudon Extragalactic Database, see Paturel et al. 1988) and Hypercat (Prugniel \& Simien 1996) databases. We only kept $\sim 2.6$ million of these objects that are identified as either galaxies or quasars, omitting stars and nebulae. Distance moduli from spectroscopic redshift measurements are given for each entry in HyperLEDA, with a 36 percent corresponding mean error of luminosity distances.

The 2MASS XSC catalogue consists of $\sim 1.6$ million objects, each having coordinates and photometric magnitude data in the $J, H$ and $K_{s}$ infrared bands. Sources with apparent magnitude $K_{S}<13.5^{m}$ (i.e. $\sim 50$ percent of the sources) are classified as galaxies with 98 percent confidence (Jarrett et al. 2000), nevertheless we included all 2MASS XSC objects in our cross-matching process, even the ones not satisfying this criterion. Since 2MASS XSC objects are all extended sources beyond the point spread function, and classification tests performed by Skrutskie et al. (2006) implemented filters to exclude double and triple stars, we expect that the stellar contamination of the whole sample is negligible. Since 2MASS is an infrared survey, $B$ magnitudes and redshifts (both of which are important parameters for EM follow-up target selections) are not included in the 2MASS XSC catalogue. Despite the lack of these parameters, this catalogue is an important source of infrared magnitudes, which can be used for estimating stellar masses of galaxies (Bell et al. 2003) and thus as an alternative tracer of BNS mergers (see e.g. Fong et al. 2013 and Hanna et al. 2014).

Authors of Bilicki et al. (2014) constructed the 2MPZ catalogue by cross-matching 2MASS XSC with the Widefield Infrared Survey Explorer catalogue (Wright et al. 2010) and the SuperCOSMOS optical catalogue (Hambly et al. 2001). 2MPZ contains both $B$ magnitudes and photometric redshifts for its more than 900,000 galaxy entries. Bilicki et al. computed photometric redshifts with an artificial neural network algorithm trained on redshift surveys. Errors of these photometric redshifts are not given for the specific entries, but they are nearly independent of distance, and have an all-sky average of $\Delta z=1.5 \times 10^{-2}$. Spectroscopic

\footnotetext{
7 vizier.u-strasbg.fr/
} 
redshifts are also available for approximately $\sim 300,000$ entries, with an average error of $\Delta z=1.5 \times 10^{-4}$. Each galaxy's $B$ magnitude has its own estimated error, with an average of $\Delta B=0.06^{m}$. We have replaced photometric redshifts of $\sim 40,000$ galaxies in $2 \mathrm{MPZ}$ with spectroscopic redshifts from the 2MASS Redshift Survey catalogue ${ }^{8}$ (Huchra et al. 2012).

The SDSS-DR12Q catalogue contains $\sim 300,000$ spectroscopically targeted and visually confirmed quasars from the Baryon Oscillation Spectroscopic Survey of the Sloan Digital Sky Survey III (Dawson et al. 2013). All of these quasars have photometric redshift values and corresponding errors associated to them.

We found it crucial to reduce the level of redundancy in our catalogue by eliminating duplicates. In order to achieve this goal, we applied a $k$-dimensional tree method (Bentley 1975), which builds a space-partitioning data structure, where a nearest neighbour search in parameter space can be done effectively with an $\mathscr{O}(\log N)$ time complexity. When matching two selected catalogues, we identified several duplicates by simply matching their object names. For all objects with common names, we calculated the differences between their RA, dec, $B$ magnitude, and $d_{L}$ values. After confirming empirically that all four sets of differences are normally distributed, we measured the corresponding standard deviations (see Table 1), and applied a chi-squared test to the galaxies that are not part of the exactly matchedby-name set. The degrees of freedom in the chi-squared tests depended on the number of parameters from the set of four (RA, dec, $B$, and $d_{L}$ ) that the matched entries had in common. A threshold of 99 percent was used in the chi-squared test, which means that only 1 percent of duplicates were falsely identified as being two different objects.

The advantage of this cross-matching method is that it can differentiate between galaxies residing in close proximity of each other by chance, but having significantly different redshifts and/or $B$ magnitudes. The number of accidental proximities of different galaxies rise with the number of entries in the merged catalogues, which makes it necessary to use more parameters beyond sky coordinates in the matching process. We first cross-matched GWGC with HyperLEDA, and 2MASS XSC with 2MPZ (where both catalogues use the same notation for the objects, so the duplicates have been identified simply by matching the object names), and then cross-matched the two resulting catalogues. As we found no objects matching between this catalogue and SDSS-DR12Q, we simply merged these two catalogues with each other.

Cross-matching the five catalogues with our method resulted with 2,965,718 galaxies, 297,014 quasars and 149 globular clusters (i.e. a total of $3,262,881$ objects) in the GLADE catalogue. The sky distribution of all GLADE objects is shown in Figure 1 as a number density plot. The main reason of anisotropy in the distribution of GLADE objects is the different sensitivity of surveys and the uneven number of observations made toward different sky directions (note that the densest strips in the plot correspond to the HyperLEDA catalogue). The plane of the Milky Way is also noticeable, as the gas and dust content of the disk significantly reduces the visibility of galaxies in the background at optical and
Table 1. Standard deviations of differences between RA, dec, $B$ magnitude, and luminosity distance $\left(d_{L}\right)$ values of pairs matching by their object names in two cross-matched catalogs (see Section 2 for details). We give these standard deviations, as well as the number of such pairs $(N)$ found in the two steps of (i) crossmatching GWGC with HyperLEDA (left column), and (ii) crossmatching [2MASS XSC + 2MPZ] with [GWGC + HyperLEDA] (right column).

\begin{tabular}{|c|c|c|}
\hline & $\begin{array}{c}\text { GWGC } \times \\
\text { HyperLEDA }\end{array}$ & $\begin{array}{c}{[2 \mathrm{MASS} X \mathrm{XSC}+2 \mathrm{MPZ}] \times} \\
{[\text { GWGC }+ \text { HyperLEDA }]}\end{array}$ \\
\hline$\sigma_{\mathrm{RA}}[\mathrm{deg}]$ & $1.2 \times 10^{-3}$ & $3.0 \times 10^{-4}$ \\
\hline$\sigma_{\mathrm{dec}}[\mathrm{deg}]$ & $7.9 \times 10^{-4}$ & $9.0 \times 10^{-5}$ \\
\hline$\sigma_{B}[\mathrm{mag}]$ & 0.588 & 0.320 \\
\hline$\sigma_{d_{L}}[\mathrm{Mpc}]$ & 29.1 & 93.5 \\
\hline$N$ & 28,279 & 152,894 \\
\hline
\end{tabular}

infrared wavelengths, resulting in a lower number density of GLADE objects along the galactic plane.

Redshifts of GLADE objects (where they are available) needed corrections for the objects' peculiar motions, in order to arrive at more accurate distance estimates from Hubble's law. We used the peculiar velocity field published in Carrick et al. (2015) for this correction, where peculiar velocities are defined at vertices of a uniform grid with $1.56 \mathrm{Mpc} / h$ linear resolution, within a distance limit of $\pm 200 \mathrm{Mpc} / h$. We estimated the peculiar velocity of each GLADE object using a trilinear interpolation from peculiar velocity values at the eight nearest vertices, corrected the redshift of the object with the obtained value, and repeated the same process over and over again until the result converged to a certain redshift value (we found that all redshift values successfully converged).

GLADE as a final product is available as a txt file on the GLADE public website ${ }^{9}$. Columns of each line of the file contain the following data (if available) for a single GLADE object:

1: Principal Galaxies Catalogue number

2: Name in the GWGC catalogue

3: Name in the HyperLEDA catalogue

4: Name in the 2MASS XSC catalogue

5: Name in the SDSS-DR12Q catalogue

6: Object type flag. Q: the source is from the SDSSDR12Q catalogue. C: the source is a globular cluster. G: the source is not from the SDSS-DR12Q catalogue and not identified as a globular cluster

7: Right ascension in degrees

8: Declination in degrees

9: Luminosity distance in Mpc

10: Error of luminosity distance in Mpc

11: Redshift

12: Apparent $B$ magnitude

13: Absolute error of apparent $B$ magnitude

14: Absolute $B$ magnitude

15: Apparent $J$ magnitude

16: Absolute error of apparent $J$ magnitude

17: Apparent $H$ magnitude

18: Absolute error of apparent $H$ magnitude

19: Apparent $K$ magnitude 

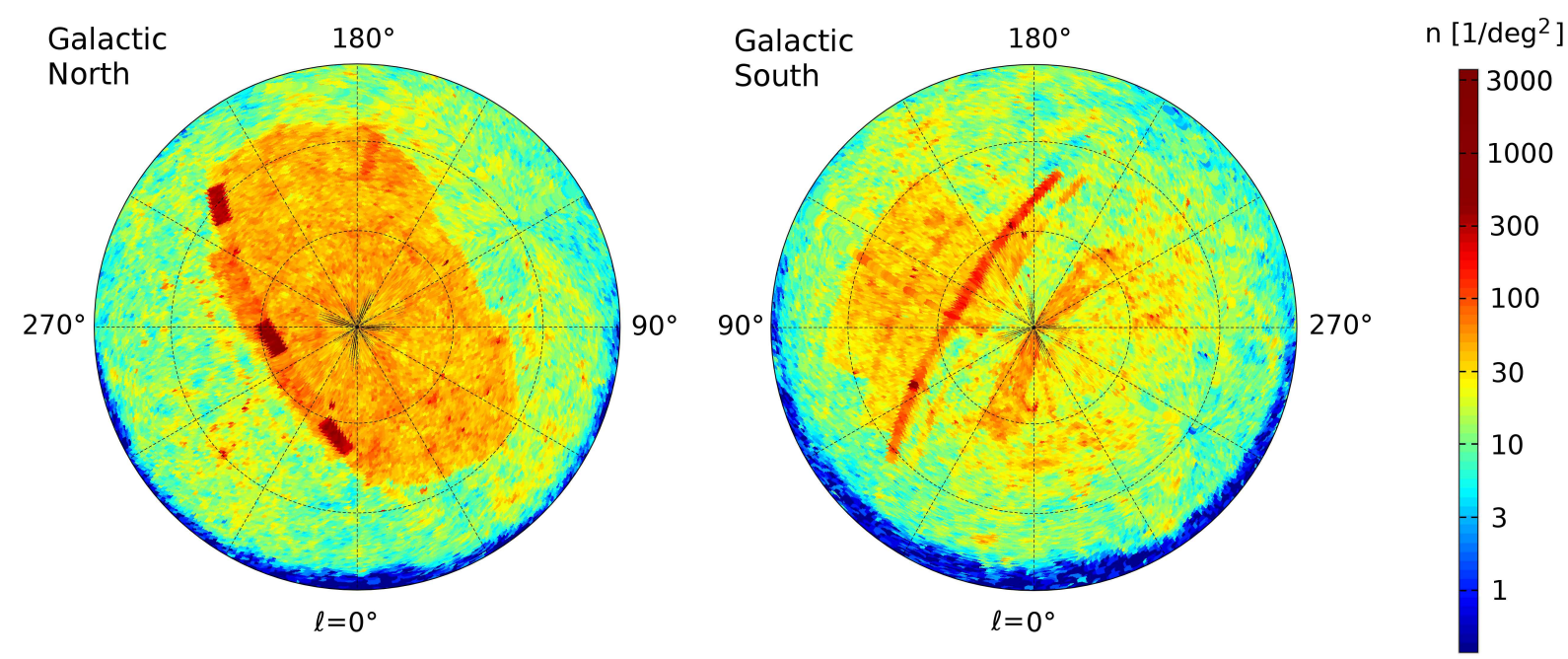

Figure 1. The number density ( $n$ ) of objects in GLADE, using azimuthal projection with galactic coordinates. The plane of the Milky Way obscures the visibility of background galaxies near the edges of the two plots. Overdense (red) patches and stripes, originating from the HyperLEDA catalogue (see Makarov et al. 2014), show up as a result of deeper, more sensitive surveys, that have been made towards the corresponding sky directions.

\section{0: Absolute error of apparent $K$ magnitude}

21: Luminosity distance measurement flag. 0 : the object has neither measured distance nor measured redshift value. 1: the object has measured redshift value from which we have calculated its distance. 2: the object has measured distance value from which we have calculated its redshift. 3: we have replaced the measured photometric redshift with spectroscopic redshift from the 2MASS Redshift Survey catalogue, from which we have calculated its distance.

22: Velocity correction flag. It indicates whether the peculiar velocity correction was not ('0') or was ('1') applied when the distance of the object was calculated.

\section{COMPLETENESS}

We quantify the completeness of GLADE with two different methods: (i) by comparing, within different luminosity distance limits, the integrated $B$ luminosity of GLADE galaxies to calculated reference values (see details in next paragraphs), and (ii) by comparing luminosity distributions of GLADE galaxies within different luminosity distance shells to the Schechter function. In this paper, we only give completeness values up to $d_{\mathrm{L}}=200 \mathrm{Mpc}$. Since there are only 2 quasars in GLADE with $d_{\mathrm{L}} \leq 200 \mathrm{Mpc}$, and only 5 percent of galaxies with $d_{\mathrm{L}} \leq 200 \mathrm{Mpc}$ not having $B$ magnitude data, we simply excluded quasars and such galaxies from our completeness measurements.

The first method we applied is the one used in White et al. (2011), which allows a direct comparison between the completeness of GWGC and of GLADE. With this method, we compared the integrated $B$-band luminosity of galaxies in GLADE up to different distance limits to the same values expected for a complete catalogue of homogeneously distributed galaxies. According to Kopparapu et al. (2008), such a complete sample of galaxies should have an average $B$-band luminosity density of $(1.98 \pm 0.16) \times 10^{-2} L_{10} \mathrm{Mpc}^{-3}$, where $L_{10}=10^{10} L_{B, \odot}$ and $L_{B, \odot}$ is the solar luminosity in the $B$-band, i.e. $L_{B, \odot}=2.16 \times 10^{33} \mathrm{erg} / \mathrm{s}$. The average $B$-band luminosity density was estimated by Blanton et al. (2003) up to $z=0.1$ (i.e. $d_{\mathrm{L}}=420 \mathrm{Mpc}$ ), however Faber et al. (2007) has showed that the same value is valid within measurement errors up to $z=0.3$ (i.e. $d_{\mathrm{L}}=1.2 \mathrm{Gpc}$ ).

In Figure 2 we show a comparison of the completeness of GLADE and GWGC based on our first completeness measure. Note that before producing this plot, we have updated all luminosity distances and $B$-band luminosities of GWGC objects where more recent measurements of these were available. As shown in Figure 2, based on this completeness measure, GLADE is complete up to $\sim 37 \mathrm{Mpc}$, has a completeness of $\sim 61$ percent within the maximal value of single-detector BNS ranges for aLIGO during O2 $(\sim 100$ $\mathrm{Mpc}), \sim 54$ percent within the minimal planned BNS range during O3, and $\sim 48$ percent within the planned BNS range of single aLIGO detectors with design sensitivity $(\sim 173 \mathrm{Mpc}$, see Barsotti et al. 2018).

A second method was used to determine the completeness of GLADE using the Schechter function with parameters $\phi^{*}=(1.6 \pm 0.3) \times 10^{-2} h^{3} \mathrm{Mpc}^{-3}, a=-1.07 \pm 0.07$, $L_{B}^{*}=(1.2 \pm 0.1) \times 10^{10} h^{-2} L_{B, \odot}($ Gehrels et al. 2016). We divided galaxies into twelve luminosity distance shells, each having a width of $\Delta d_{\mathrm{L}}=16.7 \mathrm{Mpc}$. We constructed histograms of $B$-band luminosities in GLADE for each shell, which are plotted in Figure 3, together with the corresponding Schechter functions. Figure 3 shows that as distance increases, more and more faint galaxies are missing from GLADE, while the histograms of $B$-band luminosities in GLADE exceed the Schechter functions in the first two bins due to a local overdensity of galaxies around the Milky Way.

The integration of the Schechter function by $x=L_{B} / L_{B}^{*}$ yields the following formula:

$\phi^{*} L_{B}^{*} \int_{x_{1}}^{\infty} x^{a+1} e^{-x} \mathrm{~d} x=\phi^{*} L_{B}^{*} \Gamma\left(a+2, x_{1}\right)$,

where following Gehrels et al. (2016), we have chosen $x_{1}=$ 


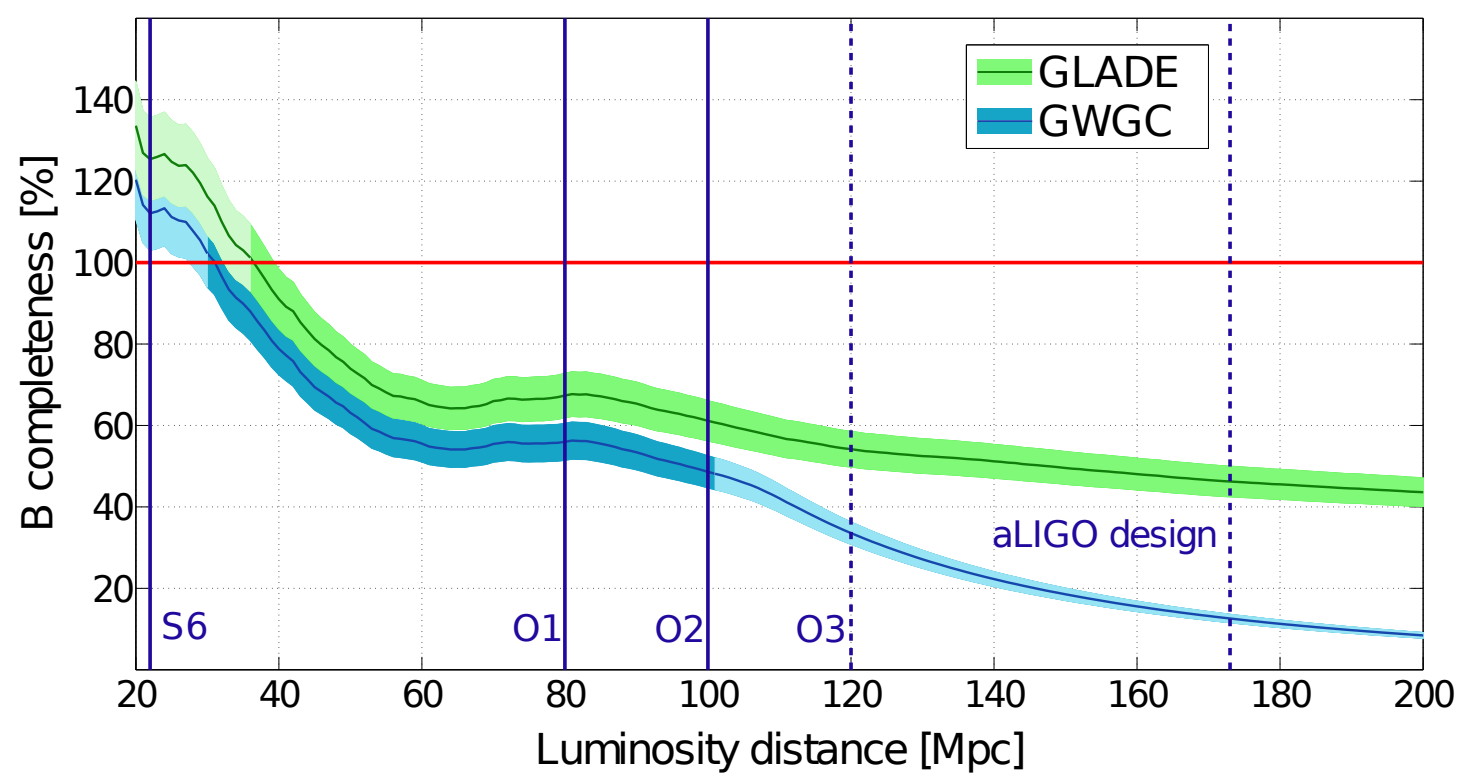

Figure 2. The plots show the normalized integrated $B$-band luminosity of galaxies in GLADE (green) and in GWGC (blue) within luminosity distances indicated on the $x$-axis. Note that before producing this plot, we have updated all luminosity distances and $B$-band luminosities of GWGC galaxies where more recent measurements were available. The normalization is carried out with the integrated $B$-band luminosity calculated from the average $B$-band luminosity density of a complete catalogue of homogeneously distributed galaxies in the $z<0.1$ local universe (see Kopparapu et al. 2008 for details). Faded segments of the curves (within 30 Mpc for GWGC, and within $37 \mathrm{Mpc}$ for GLADE) indicate the fact that completeness values exceed 100 percent within these radii due to a local overdensity of galaxies around the Milky Way within these radii. The curve for GWGC is faded over $100 \mathrm{Mpc}$ as well, since the catalogue applies a cutoff at this distance, which results in sharp decrease of its completeness (see Section 2 for details). Vertical lines represent single-detector BNS ranges at various stages of development of LIGO detectors. The solid line marked with 'S6' shows the highest BNS range value that was achieved by H1 during the sixth science run of Initial LIGO (Abadie et al. 2012c). The solid lines with the 'O1' and 'O2' labels represent the highest BNS ranges obtained by H1 during the O1 (80 Mpc) and O2 (100 Mpc) runs, respectively. The dashed lines marked with the labels 'O3' and 'aLIGO design' are the planned BNS ranges of a single aLIGO detector during O3 (at least 120 Mpc, see Abbott et al. 2016b) and at design sensitivity (173 Mpc, see Barsotti et al. 2018), respectively. Widths of the lighter green and blue stripes represent errors arising from uncertainties in the averaged $B$-band luminosity density value.

0.626, which (according to the Schechter function) is the integral bound that divides the galaxies into two subsets with equal total luminosities. We are interested in the brighter subset of these galaxies, as they would be the first targets of EM follow-up observations. In order to estimate the completeness of GLADE, we compared the integrated $B$-band luminosity of the brighter subset in each shell to the corresponding expected value calculated from the Schechter function. The completeness of GLADE in each shell is plotted in Figure 4, together with the completeness values for GWGC, the 2MASS catalogue, and the CLU catalogue, from Gehrels et al. (2016).

\section{APPLICATIONS OF GLADE}

The purpose of GLADE is to (i) help identifications of host candidates for GW events, (ii) support target selections for EM follow-up observations of GW candidates, (iii) provide input data on the matter distribution of the local universe for astrophysical or cosmological simulations, and (iv) help identifications of host candidates for poorly localised EM transients, such as GRBs observed with the IPN. In this section we show how GLADE has already been used in these areas by several collaborations.

GLADE has been used in identifications of host candi- dates and in target selections for EM follow-up observations of GW events by multiple collaborations. Following the detection of the first GW signal from a coalescencing BNS (GW170817, see Abbott et al. 2017c) an extensive observing campaign was launched, which led to the identification of an EM counterpart by multiple observer partners of the LIGO-Virgo Collaboration (Abbott et al. 2017b). Several of these teams used GLADE to maximize the chance of detecting the counterpart. For example, Arcavi et al. (2017) found the optical counterpart with the Las Cumbres Observatory global network of robotic telescopes by targeting specific galaxies within the localisation area chosen from GLADE. Castro-Tirado et al. (2017) used the JGT robotic telescope at the BOOTES-5 station to image 15 galaxies from GLADE, resulting in finding the optical counterpart in the outskirts of NGC 4993. Grado et al. (2017) used the GWsky tool to generate a pointing for the VLT Survey Telescope around the maximum probability pixel, however NGC 4993 was not inside this pointing. GLADE is used as an input in GWsky, which is an interactive tool that tiles the localisation area of a GW event based on specific telescope parameters (Greco et al. 2018 in prep.) ${ }^{10}$.

10 An additional web-based tool that uses GLADE as an input is Skymap Viewer, which interactively shows skymaps and host 


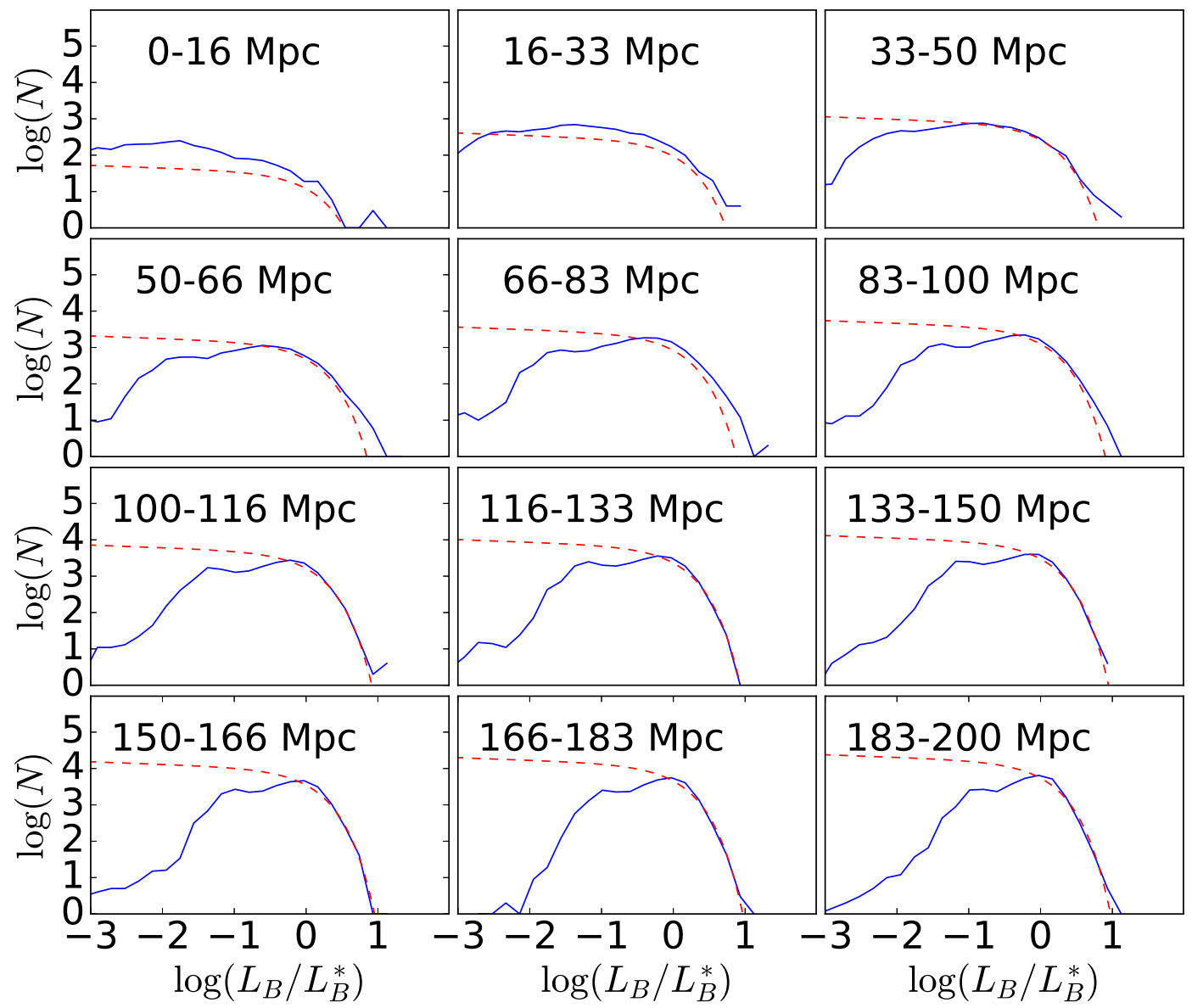

Figure 3. Luminosity histograms of GLADE galaxies within different distance shells in terms of their measured $B$-band luminosities (blue solid line), compared to the same histograms we expect for complete catalogues based on $B$-band Schechter function measurements (red dashed line). We constructed this figure in a way to allow a direct comparison with the histograms shown in Figure 2 of Gehrels et al. (2016) for the Census of the Local Universe catalogue. $L_{B}^{*}=(1.2 \pm 0.1) \times 10^{10} h^{-2} L_{B, \odot}$ is the characteristic luminosity of the Schechter function.

Note that GWsky is used by the GRAWITA collaboration (Brocato et al. 2017) as well. The Pi of the Sky robotic telescope surveyed an area that partially overlapped with the initial localisation area of GW170817, and Batsch et al. (2017) searched for transients connected with objects from GLADE, however the telescope did not target NGC 4993, and thus the counterpart was not found. The HESS collaboration (de Naurois 2017), which operates the High Energy Stereoscopic System investigating cosmic gamma rays, carried out follow-up observations with three pointings derived from the initial LIGO skymap that was cross-correlated in three dimensions with GLADE. The first of these three pointings covered NGC 4993, which region they continued to monitor the following nights as well, however, their preliminary analysis did not reveal significant gamma-ray emission.

galaxy candidates for GW events (see Li \& Williams 2016 and losc.ligo.org/s/skymapViewer/).
The multi-messenger observing of GW170817 allowed its use in measuring the local expansion rate of the universe, characterised by the Hubble constant (Abbott et al. 2017d, for its theoretical background see Schutz 1986 and Del Pozzo 2012). This was carried out by assuming NGC 4993 (the host of the EM counterpart, see Abbott et al. 2017b) to be the host of the GW source producing GW170817. A similar analysis can be carried out using data only from the GW observation itself. In such a calculation, a prior on the redshift can be obtained from galaxies inside the localisation volume, but it must be corrected for incompleteness of the galaxy sample. GLADE would be an ideal tool for this due to its high completeness and due to the fact that it directly contains all relevant galaxy data that is necessary for this type of an analysis.

We continuously use GLADE in identifying potential host galaxies for GRBs detected and poorly localised by the IPN. The identification process starts automatically whenever an IPN $3 \sigma$ localisation error box for a GRB is published 


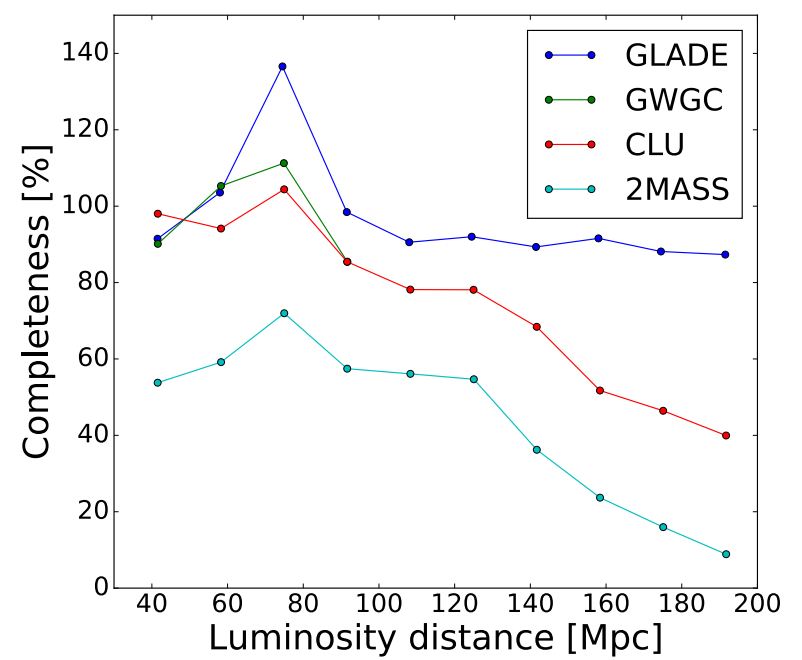

Figure 4. Completeness in various distance bins (having a 16 Mpc width) of four different galaxy catalogues relative to the Schechter function for the brighter half of galaxies. Data for the GWGC, CLU, and 2MASS catalogues are taken directly from Gehrels et al. (2016), and for GLADE it has been calculated using the same method. It can be seen that GLADE contains all the bright galaxies giving half of the total $B$ luminosity up to $d_{L}=91 \mathrm{Mpc}$, and even at larger distances GLADE has a higher completeness than the other three catalogues. Note, that we used only the brighter half of all the galaxies for producing this figure, so even if a catalogue has 100 percent completeness, it can lack dimmer galaxies. Also note, that the peak visible in the $66-83$ $\mathrm{Mpc}$ bin for all four completeness curves is due to the presence of the Perseus-Pisces Supercluster (Gregory et al. 1981) within this bin.

on the GCN Circular website ${ }^{11}$. The process identifies galaxies inside the error box, along with those residing in close proximity of it, to account for the possibility of a pair of neutron stars being kicked out from its host galaxy, and enter the error box before they merge and produce a short GRB. According to Fong \& Berger (2013), 95\% of all BNSs kicked out from a galaxy should merge within a $100 \mathrm{kpc}$ range around their hosts, therefore we decided to use this as our spatial offset limit. Our code shows the identified host candidates in the form of a table, and lists their luminosity distances, their angular distances from the closest point of the error box, their projected distances from the error box, and the probability that a BNS kicked out from the galaxy reaches the aforementioned projected distance before the merger. We calculated the probability using the observed distribution of projected physical offsets of short GRBs from their host galaxies, shown in Fig. 5 of Fong \& Berger (2013). Note, that in order to have a BNS coalescence inside the error box with this probability, one must assume that the kick occurred towards the closest point of the error box from the galaxy center. A skymap is also created showing the $\pm 0.2^{\circ}$ region around the error box with all GLADE galaxies indicated differently depending on the source of their distance data. Results of the analyses for different individual GRBs can be accessed directly on the GLADE website ${ }^{12}$.

Our analysis for GRB 150906B was of particular interest due to its proximity to a group of nearby galaxies identified as potential hosts (Abbott et al. 2016d). The localised sky region for GRB 150906B (Hurley et al. 2015) lies close to NGC 3313 which has a luminosity distance of $54 \mathrm{Mpc}$, a distance that is well within the BNS range of a single aLIGO detector. Our pipeline has found one GLADE galaxy inside the error box and another one having an angular distance of 16.62 " and a projected distance of 40-52 kpc from the closest point of the GRB error box. The two galaxies have a luminosity distance of $536 \pm 75 \mathrm{Mpc}$ and $559 \pm 75 \mathrm{Mpc}$, respectively. These distances are more compatible with predictions given by Ruffini et al. (2015) and Zhang, Zhang \& Zhang $(2015)$ based on $E_{\mathrm{p}}-E_{\text {iso }}$ and $E_{\mathrm{p}}-L_{\text {iso }}$ relations for short GRBs $(z \sim 0.1)$ than the galaxies of the NGC 3133 group, making the two galaxies far more probable hosts.

\section{CONCLUSIONS AND FUTURE WORK}

The GLADE catalogue is a value-added full-sky catalogue containing more than 3.26 million objects from which 2.97 million are categorized as galaxies, and the rest as quasars or globular clusters. It is complete up to $d_{L}=37_{-4}^{+3} \mathrm{Mpc}$ in terms of cumulative $B$ luminosity of galaxies within distance $d_{L}$, and contains all of the bright galaxies giving half of the total $B$ luminosity up to $d_{L}=91 \mathrm{Mpc}$. This high value of completeness and the presence of $B$ magnitudes and distances for most of the entries can make GLADE a useful tool for the identification of host candidates for GW events, for supporting target selections for EM follow-up observations of GW candidates, as well as for a handful of other purposes for the broader astronomical community. GLADE has already been used in several different projects, e.g. in finding the optical counterpart of GW170817, the first GW signal from a BNS coalescence, and in identifying potential host galaxies for poorly localised GRBs.

We are continuously improving GLADE in order to keep its suitability for EM follow-up efforts in the future. We are currently working on matching additional galaxy catalogues (such as WISE and Pan-STARRS) with GLADE in order to improve its completeness. Furthermore, we seek ways to develop the catalogue to include other relevant parameters, such as stellar mass and BNS formation rate estimates for the individual galaxies. Since the number density of GLADE objects is anisotropic (see Figure 1), it may also be beneficial to provide completeness information constrained to different (or all) patches in the sky. We leave this task for future works on the framework of calculating catalogue completeness in 3D localisation volumes of individual GW events.

\section{ACKNOWLEDGMENTS}

This paper was reviewed by the LIGO Scientific Collaboration under LIGO Document P1800062. The authors would like to thank Bence Bécsy, Eric Chassande-Mottin, Dániel 
Erdei, Giuseppe Greco and Ákos Szölgyén for fruitful discussions throughout the project. The authors thank Christopher Berry, Maciej Bilicki and Erik Katsavounidis for their useful comments on the manuscript. This project has been supported by the Hungarian National Research, Development and Innovation Office - NKFIH K-115709 and NN 114560. PR is supported through the ÚNKP-17-4 New National Excellence program of the Ministry of Human Capacities. The authors also gratefully acknowledge the Science and Technology Facilities Council of the United Kingdom. $\mathrm{CM}$ is supported by the Science and Technology Research Council (grant No. ST/ L000946/1). We are grateful for the Wide Field Astronomy Unit (WFAU) for providing the $2 \mathrm{MPZ}$ data used in creating GLADE.

\section{REFERENCES}

Aab, A., Abreu, P., Aglietta, M., et al. 2016, Phys. Rev. D, 94, 122007

Aasi, J., Abadie, J., Abbott, B. P., et al. 2014a, ApJS, 211, 7

Aasi, J., Abbott, B. P., Abbott, R., et al. 2014b, Physical Review Letters, 113, 011102

Aasi, J., Abbott, B. P., et al. 2015, Classical and Quantum Gravity, 32, 074001

Abadie, J., Abbott, B. P., Abbott, R., et al. 2012a, A\&A, 541, A155

Abadie, J., Abbott, B. P., Abbott, R., et al. 2012b, A\&A, 539, A124

Abadie, J., Abbott, B. P., Abbott, R., et al. 2012c, arXiv: 1203.2674

Abbott, B. P., Abbott, R., Abbott, T. D., et al. 2016a, Physical Review X, 6, 041015

Abbott, B. P., Abbott, R., Abbott, T. D., et al. 2016b, Living Reviews in Relativity, 19, 1

Abbott, B. P., Abbott, R., Abbott, T. D., et al. 2016c, ApJ, 826, L13

Abbott, B. P., Abbott, R., Abbott, T. D., et al. 2016d, arXiv:1611.07947

Abbott, B. P., Abbott, R., Abbott, T. D., et al. 2017, Physical Review Letters, 119, 141101

Abbott, B. P., Abbott, R., Abbott, T. D., et al. 2017a, ApJ, 848, L12

Abbott, B. P., Abbott, R., Abbott, T. D., et al. 2017b, Physical Review Letters, 119, 161101

Abbott, B. P., Abbott, R., Abbott, T. D., et al. 2017, Nature, 551,85

Acernese, F., Agathos, M., Agatsuma, K., et al. 2015, Classical and Quantum Gravity, 32, 024001

Arcavi, I., Hosseinzadeh, G., Howell, D. A., et al. 2017, Nature, 551,64

Baret, B., Bartos, I., Bouhou, B., et al. 2012, Phys. Rev. D, 85, 103004

Barsotti, L., McCuller, L., Evans, M., Fritschel, P. 2018, LIGOT1800044-v4 Technical Note

Bartos, I., Crotts, A. P. S., \& Márka, S. 2015, ApJ, 801, L1

Batsch, Castro-Tirado, A. J., Czyrkowski, H. 2017, GCN Circular 21931

Bécsy, B., Raffai, P., Cornish, N. J., et al. 2017, ApJ, 839, 15

Bell, E. F., McIntosh, D. H., Katz, N., \& Weinberg, M. D. 2003, ApJS, 149, 289

Bentley, J. L. 1975, Commun. ACM, 18, 9

Berry, C. P. L., Mandel, I., Middleton, H., et al. 2015, ApJ, 804, 114

Bilicki, M., Jarrett, T. H., Peacock, J. A., Cluver, M. E., \& Steward, L. 2014, ApJS, 210, 9
Blanton, M. R., Hogg, D. W., Bahcall, N. A., et al. 2003, ApJ, 592,819

Brocato, E., Branchesi, M., Cappellaro, E., et al. 2017, arXiv: 1710.05915

Carrick, J., Turnbull, S. J., Lavaux, G., \& Hudson, M. J. 2015, MNRAS, 450, 317

Castro-Tirado A. J., Tello J. C., Hu Y. et al. 2017, GCN 21624

Chassande-Mottin, E., Hendry, M., Sutton, P. J., \& Márka, S. 2011, General Relativity and Gravitation, 43, 437

Coughlin, M. W., Tao, D., Chan, M. L., et al. 2018, arXiv: 1803.02255

Cowperthwaite, P. S., Berger, E., Soares-Santos, M., et al. 2016, ApJ, 826, L29

Dawson, K. S., Schlegel, D. J., Ahn, C. P., et al. 2013, AJ, 145, 10

Del Pozzo, W. 2012, Phys. Rev. D, 86, 043011

Del Pozzo, W., Berry, C., Ghosh, A., Haines, T., \& Vecchio, A. 2018, arXiv:1801.08009

Essick, R., Vitale, S., Katsavounidis, E., Vedovato, G., \& Klimenko, S. 2015, ApJ, 800, 81

Evans, P. A., Osborne, J. P., Kennea, J. A., et al. 2016, MNRAS, 455,1522

Faber, S. M., Willmer, C. N. A., Wolf, C., et al. 2007, ApJ, 665, 265

Fan, X., Messenger, C., \& Heng, I. S. 2014, ApJ, 795, 43

Finn, L. S., \& Chernoff, D. F. 1993, Phys. Rev. D, 47, 2198

Fong, W., Berger, E., Chornock, R., et al. 2013, ApJ, 769, 56

Fong, W., \& Berger, E. 2013, ApJ, 776, 18

Gehrels, N., Cannizzo, J. K., Kanner, J., et al. 2016, ApJ, 820, 136

Grado, A., Cappellaro, E., Greco, G., et al. 2017, GCN Circular 21598

Greco, G., Branchesi, M., Stratta, G., et al., in prep.

Gregory, S. A., Thompson, L. A., \& Tifft, W. G. 1981, ApJ, 243, 411

Hambly, N. C., MacGillivray, H. T., Read, M. A., et al. 2001, MNRAS, 326, 1279

Hanna, C., Mandel, I., \& Vousden, W. 2014, ApJ, 784, 8

Huchra, J. P., Macri, L. M., Masters, K. L., et al. 2012, ApJS, 199, 26

Hurley, K., Mitrofanov, I. G., Golovin, D., et al. 2013, EAS Publications Series, 61,459

Hurley K. et al., 2015, GCN Circular 18258

Iyer, B., Souradeep, T., Unnikrishnan, C. S., et al. 2011, LIGO Document M1100296

Jarrett, T. H., Chester, T., Cutri, R., et al. 2000, AJ, 119, 2498

Karachentsev, I. D., Karachentseva, V. E., Huchtmeier, W. K., \& Makarov, D. I. 2004, AJ, 127, 2031

Kasen, D., Badnell, N. R., \& Barnes, J. 2013, ApJ, 774, 25

Kopparapu, R. K., Hanna, C., Kalogera, V., et al. 2008, ApJ, 675, 1459

Li, L.-X., \& Paczyński, B. 1998, ApJ, 507, L59

Li, K., \& Williams, R. 2016, arXiv:1611.00790

Makarov, D., Prugniel, P., Terekhova, N., Courtois, H., \& Vauglin, I. 2014, A\&A, 570, A13

Metzger, B. D., Martínez-Pinedo, G., Darbha, S., et al. 2010, MNRAS, 406, 2650

de Naurois, M. 2017, GCN Circular 21674

Ochsenbein, F., Bauer, P., \& Marcout, J. 2000, A\&AS, 143, 23

Pâris, I., Petitjean, P., Ross, N. P., et al. 2016, arXiv:1608.06483

Paturel, G., Bottinelli, L., Fouque, P., \& Gouguenheim, L. 1988, European Southern Observatory Conference and Workshop Proceedings, 28, 435

Phinney, E. S. 1991, ApJ, 380, L17

Prugniel, P., \& Simien, F. 1996, A\&A, 309, 749

Ruffini R. et al., 2015, GCN Circular 18296

Schutz, B. F. 1986, Nature, 323, 310

Singer, L. P., Price, L. R., Farr, B., et al. 2014, ApJ, 795, 105 
Singer, L. P., Chen, H.-Y., Holz, D. E., et al. 2016, ApJ, 829, L15

Skrutskie, M. F., Cutri, R. M., Stiening, R., et al. 2006, AJ, 131, 1163

Somiya, K. 2012, Classical and Quantum Gravity, 29, 124007

Soneira, R. M., \& Peebles, P. J. E. 1978, AJ, 83, 845

Tanvir, N. R., Levan, A. J., Fruchter, A. S., et al. 2013, Nature, 500,547

Tully, R. B. 1987, ApJ, 321, 280

Tully, R. B., Rizzi, L., Shaya, E. J., et al. 2009, AJ, 138, 323

White, D. J., Daw, E. J., \& Dhillon, V. S. 2011, Classical and Quantum Gravity, 28, 085016

Wright, E. L., Eisenhardt, P. R. M., Mainzer, A. K., et al. 2010, AJ, 140, 1868-1881

Zhang F., Zhang B., \& Zhang B., 2015, GCN Circular 18298

This paper has been typeset from a $\mathrm{T}_{\mathrm{E}} \mathrm{X} / \mathrm{LAT}_{\mathrm{E}} \mathrm{X}$ file prepared by the author. 